\title{
Erratum to: Perceived messages about bone health after a fracture are not consistent across healthcare providers
}

\author{
Joanna E. M. Sale · Gillian Hawker · Cathy Cameron • \\ Earl Bogoch · Ravi Jain • Dorcas Beaton · Susan Jaglal • \\ Larry Funnell
}

Published online: 25 July 2014

(C) Springer-Verlag Berlin Heidelberg 2014

\section{Erratum to: Rheumatol Int}

DOI 10.1007/s00296-014-3079-y

Unfortunately, the opening sentence of the article was incorrect in the published version. The correct sentence should read:

Our purpose was to examine messages perceived by members of an osteoporosis (OP) patient group from various healthcare providers regarding bone health.

The online version of the original article can be found under doi:10.1007/s00296-014-3079-y.

J. E. M. Sale $(\varangle) \cdot$ C. Cameron · D. Beaton

Mobility Program Clinical Research Unit, Li Ka Shing

Knowledge Institute, St. Michael's Hospital, 30 Bond Street,

Toronto, ON M5B 1W8, Canada

e-mail: salej@smh.ca

J. E. M. Sale · G. Hawker · D. Beaton

Institute of Health Policy, Management and Evaluation,

University of Toronto, Suite 425 - 155 College Street,

Toronto, ON M5T 3M7, Canada

\section{G. Hawker}

Department of Medicine, University of Toronto, Suite RFE

3-805, 200 Elizabeth Street, Toronto, ON M5G 2C4, Canada

G. Hawker

Osteoporosis Research Program, Women's College Hospital,

76 Grenville Street, Toronto, ON M5S 1B1, Canada

\author{
E. Bogoch \\ Mobility Program, St. Michael's Hospital, 30 Bond Street, \\ Toronto, ON M5B 1W8, Canada \\ E. Bogoch \\ Department of Surgery, University of Toronto, 149 College \\ Street, 5th Floor, Toronto, ON M5T 1P5, Canada \\ R. Jain $\cdot$ L. Funnell \\ Osteoporosis Canada, 1090 Don Mills Road, Suite 301, \\ Toronto, ON M3C 3R6, Canada \\ S. Jaglal \\ Department of Physical Therapy, University of Toronto, \\ 160 - 500 University Avenue, Toronto, ON M5G 1V7, Canada
}

